\title{
Rede Nacional de Cuidados Continuados e Tempo de Internamento dos Doentes com Acidente Vascular Cerebral 2010-2011
}

\author{
National Post Hospital Care Project and Length of Hospitalization of \\ Patients with Stroke 2010 - 2011
}

Catarina CANHA ${ }^{1}$, Ana MASSANO ${ }^{2}$, Hélder ESPERTO ${ }^{1}$, Joana RATO¹, Teresa FERREIRA ${ }^{3}$, Argemiro GERALDO $^{2,3}$
Acta Med Port 2013 Nov-Dec;26(6):683-688

\section{RESUMO}

Introdução: A Rede Nacional de Cuidados Continuados Integrados surgiu para proporcionar a continuação de cuidados após internamento ou para pessoas funcionalmente dependentes. Actualmente há uma grande dificuldade na integração dos doentes no domicílio ou em estruturas de retaguarda. Pretende-se comparar o impacto da referenciação para a Rede Nacional de Cuidados Continuados Integrados versus alta para domicílio, no tempo de internamento dos doentes com Acidente Vascular Cerebral, entre 2010 e 2011.

Material e Métodos: Estudo retrospectivo envolvendo os doentes internados na Neurologia A e Unidade de Acidente Vascular Cerebral dos Hospitais da Universidade de Coimbra, naquele anos. Analisaram-se 1209 processos, incluídos 819, caracterizados demograficamente, tempo de internamento, Score de Rankin modificado e destino pós-alta. Compararam-se os dados, relativamente ao tempo de internamento dos doentes com Acidente Vascular Cerebral, referenciados para a Rede Nacional de Cuidados Continuados Integrados e os com alta para domicílio, atendendo ao Score de Rankin final.

Resultados: Em 2011, aumentaram os doentes referenciados para a Rede Nacional de Cuidados Continuados Integrados, $23,5 \%$ comparativamente $21,4 \%$. Em 2011 o tempo de internamento, para um mesmo Score de Rankin, da população referenciada mantevese superior: para um Rankin de 1: 11 dias para domicílio, 26 dias para os doentes referenciados; para um Rankin de 2: 13 dias para domicílio, 29 dias para a rede; para Rankin de 3: 13 dias para domicílio, 23 dias para referenciados; para um Rankin de 4: 17 dias para domicílio, 33 dias para Rede; e para um Rankin de 5: 27 dias para domicílio, 39 dias para Rede. Comparando com os tempos de internamento da população com alta para domicílio, estima-se que tenha representado mais 1718 dias de internamento, em 2010 e 1198 dias, em 2011.

Conclusão: A Rede Nacional de Cuidados Continuados Integrados é incapaz de responder às necessidades actuais, embora o tempo de espera tenha reduzido, devido ao aumento do número de camas e da possibilidade dos doentes aguardarem vaga no domicílio. Palavras-chave: Acidente Vascular Cerebral; Admissão do Doente; Alta do Doente; Índice de Gravidade de Doença; Recuperação de Função.

\section{ABSTRACT}

Introduction: The National Post Hospital Care Project was created to provide a continuity of care after hospitalization or to functionally dependent people. Currently there is a great difficulty in the integration of patients. The objective of this paper is to compare the impact of the referral to the Project versus being discharged home, in the length of stay of stroke patients between 2010 and 2011.

Material and Methods: Retrospective study of patients admitted to the Neurology Infirmary A and Stroke Unit of Coimbra's University Hospital, in 2010 and 2011. The cases analyzed were 1 209, featuring demographic data, length of stay, Rankin Score (mRS) and destination after discharge. The data was analyzed comparing the two years concerning the length of stay of stroke patients referred to the Project and those discharged home, given the their Rankin Score.

Results: In 2011, the number patients referred to the National Post Hospital Care Project was higher, $23.5 \%$ compared to $21.4 \%$. The length of stay for the same Rankin Score of the patients referred to National Post Hospital Care Project, remained higher than those discharged home: for a Rankin Score of 1: 11, versus 26 days for the Project; Rankin Score 2: 13, versus 29 days for the project; Rankin Score 3: 13, versus 23 days for the Project; Rankin Score 4: 17, to 33 days for the Project, Rankin Score 5: 27, versus 39 days to the Project. After comparison between the length of stay of patient discharged of and those referred to the National Post Hospital Care Project, it was estimated that the referral represented an hospitalization excess of 1718 days in 2010 and 1198 days in 2011.

Conclusion: The National Post Hospital Care Project is unable to meet the actual needs although the waiting time has reduced, possibly due to the increased number of beds and the possibility of patients waiting at home.

Keywords: Patient Admission; Patient Discharge; Recovery of Function; Severity of Illness Index; Stroke.

\section{INTRODUÇÃO}

A população portuguesa tem vindo a apresentar um aumento da esperança média de vida, bem como uma redução da natalidade, resultando no envelhecimento populacional. Numa população mais idosa há uma maior prevalência de doenças crónicas, que apresentam períodos de agudização e que conferem risco acrescido para novos eventos agudos. Esta realidade exige que consigamos articular uma resposta que permita que o aumento da espe- rança média de vida seja acompanhado da manutenção de qualidade de vida, evite a sobrelotação dos hospitais de agudos com doentes que já não necessitam deste nível de cuidados, prepare os cuidadores para se tornarem capazes de fornecer os cuidados necessários, participando na reabilitação ou prevenção da perda de funcionalidade.

Reconhecendo esta necessidade foi criada em 2006 a Rede Nacional de Cuidados Continuados Integrados

1. Serviço de Medicina Interna. Centro Hospitalar e Universitário de Coimbra. Coimbra. Portugal.

2. Serviço de Neurologia. Centro Hospitalar e Universitário de Coimbra. Coimbra. Portugal.

3. Equipa de Gestão de Altas. Centro Hospitalar e Universitário de Coimbra. Coimbra. Portugal.

Recebido: 02 de Fevereiro de 2013 - Aceite: 23 de Junho de 2013 | Copyright @ Ordem dos Médicos 2013 
Tabela 1 - Caracterização das Diferentes Tipologias das Unidades de Internamento da RNCCI ${ }^{1,2}$

\begin{tabular}{|c|c|}
\hline Tipologia & Objectivo / Caracterização \\
\hline Convalescença & $\begin{array}{l}\text { - Promover a reabilitação e a independência dos utentes; } \\
\text { - Contribuir para a gestão das altas dos hospitais de agudos; } \\
\text { - Evitar a permanência desnecessária nos serviços dos hospitais de agudos; } \\
\text { - Optimizar a utilização de unidades de internamento de média e longa duração. }\end{array}$ \\
\hline Média duração e Reabilitação & $\begin{array}{l}\text { - Evitar permanências desnecessárias em hospitais de agudos; } \\
\text { - Contribuir para a gestão das altas dos hospitais de agudos; } \\
\text { - Reduzir a utilização desnecessária de unidades de internamento de convalescença e de } \\
\text { longa duração; } \\
\text { - Promover a reabilitação e a independência dos utentes. }\end{array}$ \\
\hline Longa duração e Manutenção & $\begin{array}{l}\text { - Facilitar a gestão das altas dos hospitais de agudos; } \\
\text { - Promover a autonomia e a satisfação de necessidades sociais dos doentes }\end{array}$ \\
\hline Paliativos & $\begin{array}{l}\text { - Acompanhamento, tratamento e supervisão clínica de doentes em situação clínica } \\
\text { complexa e de sofrimento decorrente de doença severa e/ou avançada, incurável e } \\
\text { progressiva. }\end{array}$ \\
\hline
\end{tabular}
progressiva.

(RNCCI) conforme decreto-lei $n^{\circ}$ 101/2006. DR 109 Serie I-A de 2006-06-06, ${ }^{1}$ onde são definidos os cuidados continuados como '... o conjunto de intervenções sequenciais de saúde e ou de apoio social, decorrente de avaliação conjunta, centrado na recuperação global entendida como o processo terapêutico e de apoio social, activo e contínuo, que visa promover a autonomia melhorando a funcionalidade da pessoa em situação de dependência, através da sua reabilitação, readaptação e reinserção familiar e social'. Neste documento são também definidos os objectivos específicos da rede que se centram na melhoria das condições de vida e de bem-estar das pessoas em situação de dependência, incluindo o apoio aos familiares ou prestadores informais, na respectiva qualificação e na prestação dos cuidados, devendo ser incentivados a participar e ser co-responsabilizados. ${ }^{1}$

A RNCCl engloba várias vertentes: unidades de internamento, unidades de ambulatório (unidade de dia e de promoção de autonomia), equipas hospitalares (equipas de gestão de altas e equipas intra-hospitalares de suporte em cuidados paliativos) e equipas domiciliárias (equipas de cuidados continuados integrados e equipas comunitárias de suporte em cuidados paliativos).

As unidades de internamento englobam quatro tipolo- gias, idealizadas com diferentes objectivos, que implicam diferentes características físicas e de constituição da equipa multidisciplinar envolvida. Na Tabela 1 encontramos caracterizadas as diferentes tipologias.

Inicialmente, o projecto de implementação das infra-estruturas necessárias para cumprir este objectivo dividia-se em três fases: a primeira, entre 2006 e 2008, previa a cobertura de $30 \%$ das necessidades existentes na população; a segunda fase, entre 2009 e 2012 que previa a cobertura de $60 \%$ e uma última fase, entre 2012 e 2016 , em que se atingiriam os $100 \%$. Posteriormente, este plano foi alterado, prevendo-se que a RNCCl esteja a funcionar a $100 \%$ em 2013. ${ }^{2}$

O objectivo principal da criação da $\mathrm{RNCCl}$ foi no fundo optimizar o tratamento dos doentes após evento agudo, ou agudização de doença crónica e proporcionar conforto aos doentes em situação terminal. Esta optimização passa também por facilitar o fluxo de doentes dos Hospitais de agudos, permitindo agilizar as altas de doentes que já não necessitem deste nível de cuidados para Unidades preparadas para os receber, ou para domicílio, uma vez assegurada a continuidade de cuidados, quer através de equipas de apoio, quer através da formação dos cuidadores informais. Deste modo promovem-se uma melhor reabilitação e 
Tabela 1 - Caracterização das Diferentes Tipologias das Unidades de Internamento da RNCCl ${ }^{1,2}$ (continuação).

\begin{tabular}{ll}
\hline Tempo de internamento previsto & Serviços \\
\hline Até 30 dias consecutivos por cada admissão; & $\begin{array}{l}\text { Cuidados médicos e de enfermagem permanentes; } \\
\text { Exames complementares de diagnóstico; } \\
\text { Prescrição e administração de fármacos; }\end{array}$ \\
$\begin{array}{l}\text { Cuidados de fisioterapia e apoio psicossocial; } \\
\text { Higiene, conforto e alimentação; } \\
\text { Convívio e lazer. }\end{array}$
\end{tabular}

Superior a 30 e inferior a 90 dias consecutivos, por cada admissão;

Superior a 90 dias consecutivos;
Cuidados médicos diários e de enfermagem permanentes;

Cuidados de fisioterapia e de terapia ocupacional;

Prescrição e administração de fármacos;

Apoio psicossocial;

Higiene, conforto e alimentação;

Convívio e lazer.

Actividades de manutenção e de estimulação;

Cuidados de enfermagem diários e cuidados médicos;

Prescrição e administração de fármacos;

Apoio psicossocial e controlo fisiátrico periódico;

Cuidados de fisioterapia e de terapia ocupacional;

Animação sócio-cultural;

Higiene, conforto e alimentação;

Apoio no desempenho das actividades da vida diária.

Cuidados médicos diários e de enfermagem permanentes;

Exames complementares de diagnóstico;

Prescrição e administração de fármacos;

Cuidados de fisioterapia;

Consulta, acompanhamento e avaliação de doentes internados em outros serviços

ou unidades;

Acompanhamento e apoio psicossocial e espiritual;

Actividades de manutenção;

Higiene, conforto e alimentação;

Convívio e lazer. reintegração, prevenindo as complicações de internamentos desnecessariamente prolongados. ${ }^{3}$

A RNCCI para assegurar a qualidade assistencial apresenta um sistema de monitorização contínua, que se expressa na forma de um relatório anual de consulta pública. Nesse relatório, avaliam-se as várias vertentes desta estrutura: a implementação da rede (números de camas disponíveis/tipologia, números de Equipas de Gestão de Alta, Equipas Coordenadoras Locais e Equipas de Cuidados Continuados Integrados); a referenciação (tempo de avaliação das propostas pelas equipas coordenadoras locais, tempo de identificação de vaga nas Equipas Coordenadoras Regionais); caracterização dos doentes referenciados (critérios de ingresso versus exclusão; doente a aguardar vaga, motivo de referenciação, dados demográficos); resultados de actividade assistencial (funcional e dependência na admissão versus alta, úlceras de pressão, quedas, avaliação da dor, óbitos); e formação (ensino a familiares ou cuidadores informais, formação profissional).

No relatório relativo ao ano de 2011, conclui-se que número de camas contratadas em funcionamento a 31 de Dezembro de 2011, cresceu $21 \%$ relativamente a 2010 , representando 970 novos lugares de internamento, sendo que o total de utentes referenciados para a Rede durante
2011 foi de 30103 utentes, evidenciando um aumento de $40,1 \%$ em relação ao acumulado de utentes referenciados desde o início da RNCCI com 105257 referenciações. Entre os motivos das propostas de referenciação de utentes para a RNCCI, 'a dependência de AVD' é a nível nacional o principal motivo de referenciação 90\% (igual em 2010), seguido de 'Ensino utente/Cuidador informal' 61\% (85\% em 2010). 4,5

A funcionalidade e qualidade da $\mathrm{RNCCl}$ são avaliadas e monitorizadas nos relatórios anuais, estando praticamente concluída a sua implementação, torna-se importante avaliar a sua capacidade de resposta a um dos seus objectivos principais: facilitar o fluxo de doentes dos Hospitais de Agudos, prevenindo os internamentos prolongados e as suas complicações. Este estudo pretende avaliar o impacto da referenciação dos doentes para a $\mathrm{RNCCl}$, nos tempos de internamento hospitalar entre o ano de 2010 e 2011. Foram analisadas as altas dos doentes com diagnóstico de internamento de Acidente Vascular Cerebral (AVC), situação que geralmente confere algum grau de dependência para AVDs, motivo pelo qual mais frequentemente foram referenciados os doentes em 2010 e 2011. Ao analisar doentes com uma mesma patologia anularam-se variáveis que poderiam enviesar os resultados e, por outro lado, permitiu 
agrupar os doentes de acordo com o seu grau de dependência.

Com este trabalho pretende-se comparar os tempos de internamento dos doentes referenciados para Unidade de Internamento, com aqueles que tiveram alta para o domicílio, entre os anos de 2010 e 2011, bem como avaliar relação entre o grau de dependência e a necessidade de referenciação para $\mathrm{RNCCl}$.

\section{MATERIAL E MÉTODOS}

Trata-se de um estudo retrospectivo envolvendo os doentes internados na Enfermaria de Neurologia A e Unidade de Acidente Vascular Cerebral, do Hospitalar Universitário de Coimbra (HUC), em 2010 e 2011. Analisaram-se 1209 processos de doentes através da consulta do processo clínico. Dos processos analisados seleccionaram-se os doentes internados por Acidente Vascular Cerebral e estes foram caracterizados demograficamente, tempo de internamento, modified Rankin Score (mRS) e destino pós-alta.

O Score de Rankin é um instrumento para avaliação do grau de dependência, validado para doentes com AVC, variando de 0 (sem sintomas) a 6 (morte) (Tabela 2).

Analisaram-se comparativamente os dados dos dois anos, relativamente ao tempo de internamento dos doentes referenciados para unidades de internamento da $\mathrm{RNCCl}$, com os doentes que tiveram alta para o domicílio.

\section{RESULTADOS}

Dos 1209 processos revistos, foram seleccionados 819 processos, que correspondem a doentes internados com diagnóstico de AVC. Durante o ano de 2010 foram internados 379 doentes com AVC, 228 homens e 151 mulheres. No ano de 2011 houve um aumento do número de internamentos, tendo sido internados 440 doentes. De um ano para outro manteve-se a prevalência de sexo masculino e população idosa, acima dos 65 anos.

Os doentes referenciados em 2010 representaram $21,4 \%(n=81)$ do total dos doentes internados com o diagnóstico de AVC. (Tabela 3) Em 2011, houve um pequeno aumento de referenciação representado $23,5 \%(n=103)$ do total dos doentes internados. Em ambos os anos observou-se uma maior necessidade de referenciação para Scores de Rankin mais elevados, ou seja, para doentes com um grau maior de dependência. Os doentes com Scores de Rankin superiores a 3 representam em 2010, 67,9 \% $(n=55)$ dos doentes referenciados e 83,5\% $(n=86)$ em 2011.

O grupo com maior necessidade de referenciação foi o que apresentava um Rankin à data de alta de 5, em ambos os anos. Em 2010 foram referenciados 53,1\% $(n=26)$ dos doentes com mRS de 5 e em 2011, 67,2\% ( $n=41)$.

Em 2010, 6 doentes do total de 81 referenciados faleceram após já terem sido referenciados. Em 2011, faleceram 14 doentes em situação idêntica, a aguardar vaga na RNCCl.

Os doentes que pertenciam a áreas de influência de outros hospitais, foram transferidos, sempre que possível, para hospitais mais próximos da sua área de residência (Tabela 4).

Relativamente ao tempo de internamento, verificou-se

Tabela 2 - Escala de Rankin Modificada

\begin{tabular}{|c|c|c|c|c|c|}
\hline Score & \multicolumn{5}{|l|}{ Descrição } \\
\hline 0 & \multicolumn{5}{|c|}{ Sem sintomas } \\
\hline 1 & \multicolumn{5}{|c|}{ Sem incapacidade significativa apesar dos sintomas; capaz de executar as actividades quotidianas habituais } \\
\hline 2 & \multicolumn{5}{|c|}{$\begin{array}{l}\text { Incapacidade ligeira; incapaz de executar todas as actividades prévias, mas capaz de executar as actividades de vida } \\
\text { diária de uma forma independente }\end{array}$} \\
\hline 3 & \multicolumn{5}{|c|}{ Incapacidade moderada; necessita de alguma ajuda de outros, mas capaz de deambular sem assistência } \\
\hline 4 & \multicolumn{5}{|c|}{$\begin{array}{l}\text { Incapacidade moderamente severa; incapaz de deambular sem ajuda e incapaz de executar as actividades de vida diária } \\
\text { sem assistência }\end{array}$} \\
\hline 5 & \multicolumn{5}{|c|}{ Incapacidade severa; restrito ao leito, incontinente e com necessidade de vigilância constante } \\
\hline 6 & \multicolumn{5}{|l|}{ Óbito } \\
\hline bela 3 & \multicolumn{5}{|c|}{ racterísticas Demográficas da População Estudada } \\
\hline \multicolumn{2}{|r|}{ Ano } & \multicolumn{2}{|c|}{$\begin{array}{c}2010 \\
(n=379)\end{array}$} & \multicolumn{2}{|c|}{$\begin{array}{c}2011 \\
(n=440)\end{array}$} \\
\hline \multirow{2}{*}{\multicolumn{2}{|c|}{$\begin{array}{l}\text { Género } \\
n(\%)\end{array}$}} & Masculino & Feminino & Masculino & Feminino \\
\hline & & $228(60,2)$ & $151(39,8)$ & $271(61,6)$ & $169(38,4)$ \\
\hline \multicolumn{2}{|c|}{ Anos (dp) } & $69(13)$ & $72(16)$ & $70(12)$ & $74(13)$ \\
\hline
\end{tabular}


Tabela 4 - Distribuição dos doentes de acordo com destino de alta e Score de Rankin

\begin{tabular}{|c|c|c|c|c|c|c|c|c|c|c|}
\hline \multirow[t]{2}{*}{ mRS } & \multicolumn{2}{|c|}{ RNCCI } & \multicolumn{2}{|c|}{ Domicílio } & \multicolumn{2}{|c|}{ Outros Serviços } & \multicolumn{2}{|c|}{ Outros Hospitais } & \multicolumn{2}{|c|}{$\begin{array}{l}\% \text { de doentes } \\
\text { por } \mathrm{mRS}\end{array}$} \\
\hline & $\begin{array}{l}2010 \\
\%(n)\end{array}$ & $\begin{array}{l}2011 \\
\%(n)\end{array}$ & $\begin{array}{l}2010 \\
\%(n)\end{array}$ & $\begin{array}{l}2011 \\
\%(n)\end{array}$ & $\begin{array}{l}2010 \\
\%(n)\end{array}$ & $\begin{array}{l}2011 \\
\%(n)\end{array}$ & $\begin{array}{l}2010 \\
\%(n)\end{array}$ & $\begin{array}{l}2011 \\
\%(n)\end{array}$ & $\begin{array}{l}2010 \\
\%(n)\end{array}$ & $\begin{array}{l}2011 \\
\%(n)\end{array}$ \\
\hline 0 & $0(0)$ & $0(0)$ & $100(37)$ & $98,2(55)$ & 0 & $1,2(1)$ & 0 & 0 & $9,8(37)$ & $12,5(55)$ \\
\hline 1 & $4,1(3)$ & $2,2(2)$ & $95,9(70)$ & $94,4(85)$ & 0 & $1,1(1)$ & 0 & $2,2(2)$ & $19,3(73)$ & $20,5(90)$ \\
\hline 2 & $11,3(8)$ & $7,2(5)$ & $83,1(59)$ & $84,0(58)$ & $1,4(1)$ & $2,9(2)$ & $4,2(3)$ & $5,8(4)$ & $19,7(71)$ & $15,7(69)$ \\
\hline 3 & $28,3(15)$ & $17,9(10)$ & $64,2(34)$ & $73,2(41)$ & 0 & $0(0)$ & $7,5(4)$ & $8,9(5)$ & $14,0(53)$ & $12,7(56)$ \\
\hline 4 & $40,4(23)$ & $62,0(31)$ & $50,9(29)$ & $18,0(9)$ & 0 & 0 & $8,8(5)$ & $20(10)$ & $15,0(57)$ & $11,4(50)$ \\
\hline 5 & $53,1(26)$ & $67,2(41)$ & $39,6(15)$ & $6,6(4)$ & 0 & $3,2(2)$ & $16,3(8)$ & $23,0(14)$ & $12,9(49)$ & $13,9(61)$ \\
\hline 6 & $15,4(6)$ & $23,7(14)$ & - & - & - & - & - & - & $10,3(39)$ & $13,4(59)$ \\
\hline Total & $21,4(81)$ & $\begin{array}{r}23,5 \\
(103)\end{array}$ & $\begin{array}{r}64,4 \\
(244)\end{array}$ & $\begin{array}{r}57,2 \\
(252)\end{array}$ & $0,3(1)$ & $1,4(6)$ & $5,3(20)$ & $8,0(35)$ & $100(379)$ & $100(440)$ \\
\hline
\end{tabular}

Tabela 5 - Tempo médio de permanência hospitalar, de acordo com modified Rankin Score (mRS).

\begin{tabular}{|c|c|c|c|c|c|c|}
\hline \multirow[t]{2}{*}{$\mathrm{mRS}$} & \multicolumn{2}{|c|}{ Domicílio } & \multicolumn{2}{|c|}{ RNCC } & \multicolumn{2}{|c|}{ Outros Hospitais } \\
\hline & $\begin{array}{c}2010 \\
\text { (dias) }\end{array}$ & $\begin{array}{c}2011 \\
\text { (dias) }\end{array}$ & $\begin{array}{c}2010 \\
\text { (dias) }\end{array}$ & $\begin{array}{c}2011 \\
\text { (dias) }\end{array}$ & $\begin{array}{c}2010 \\
\text { (dias) }\end{array}$ & $\begin{array}{c}2011 \\
\text { (dias) }\end{array}$ \\
\hline 0 & 8 & 11 & - & & - & \\
\hline 1 & 11 & 11 & 15 & 26 & - & 4 \\
\hline 2 & 10 & 13 & 42 & 29 & 9 & 13 \\
\hline 3 & 10 & 13 & 33 & 23 & 15 & 9 \\
\hline 4 & 26 & 17 & 39 & 33 & 38 & 17 \\
\hline 5 & 24 & 27 & 55 & 39 & 16 & 15 \\
\hline
\end{tabular}

que um Score de Rankin mais elevado se associa a uma permanência no hospital mais prolongada. No grupo de doentes que teve alta para domicílio, o tempo médio de internamento foi superior em 2011, à excepção dos doentes com $\mathrm{mRS}$ de 1 e de 4 . No grupo de doentes referenciados, o tempo médio de internamento diminuiu para quase todos os scores à excepção do mRS 1. Comparativamente ao tempo de internamento do grupo de doentes com alta para domicílio, os doentes referenciados permaneceram mais tempo no hospital (Tabela 5).

\section{DISCUSSÃO}

A Rede Nacional de Cuidados Continuados Integrados foi criada para colmatar uma necessidade crescente de resposta de cuidados de saúde a doentes com patologias agudas e crónicas. Esta necessidade decorre do envelhecimento populacional que se traduz no aumento do número de pessoas com este tipo de patologias. Este envelhecimento populacional espelha-se na idade média dos doentes estudados 69 anos e 70 anos do género masculino, em 2010 e 2011 respectivamente e 72 anos e 74 anos do género feminino.

O número de doentes referenciados para $\mathrm{RNCCl}$, nomeadamente para as unidades de internamento aumentou e foram referenciados, 81 doentes em 2010 e 103 doentes em 2011.

A relação entre a necessidade de referenciação e o mRS, evidencia, sem grande surpresa, que a um grau de dependência mais elevado, corresponde uma maior necessidade de referenciação. Esta situação manteve-se nos 
dois anos estudados, representando os doentes com $\mathrm{mRS}$ superiores a três, $67,9 \%(n=55)$ dos doentes referenciados em 2010 e $83,5 \%(n=86)$ dos doentes referenciados, em 2011. As famílias actualmente não estão preparadas para lidar com elevados graus de dependência, quer porque esta situação se instala como evento agudo criando uma necessidade de adaptação imediata, quer, porque sendo a população muito envelhecida, muitas vezes os cuidadores são também indivíduos já idosos, apresentando também alguma dependência. Por outro lado, os familiares mais jovens encontram-se frequentemente em idade activa, não tendo a disponibilidade para dedicarem o tempo necessário para estes doentes. Por último, as pequenas alterações que são necessárias na estrutura e organização familiar, quer de disponibilidade de cuidados, quer da reestruturação de algumas divisões da casa, implicam uma capacidade económica que muitas famílias não possuem.

Ao fazermos a comparação entre os anos de 2010 e 2011, podemos observar que os tempos de permanência no hospital dos doentes não referenciados aumentam em geral para quase todos os Scores de Rankin, excepto para o $\mathrm{mRS}$ de 4. No que diz respeito ao tempo de internamento dos doentes referenciados, estes apresentam uma situação inversa, com diminuição da permanência hospitalar para quase todos os $\mathrm{mRS}$, à excepção de $\mathrm{mRS}$ de 1. Esta situação reflecte seguramente uma melhor referenciação, um aumento de camas disponíveis na $\mathrm{RNCCl}$ e a oportunidade que surge em 2011 dos doentes com alta clínica, mas que beneficiam de internamento na $\mathrm{RNCCl}$, aguardarem no domicílio. ${ }^{6}$

Apesar da redução do tempo médio de internamento dos doentes referenciados, este manteve-se bastante superior ao tempo médio de internamento dos doentes com alta para domicílio, independentemente do mRS. Em 2011, para um $\mathrm{mRS}$ de 2, o tempo médio de internamento de um doente com alta para domicílio, foi de menos de metade (11 dias), de um doente referenciado para RNCCI (26 dias).

Com base nos dados obtidos, ao calcular o produto do número de doentes referenciados pelo tempo de internamento correspondente ao seu Rankin e, fazendo a comparação com o tempo equivalente para os doentes com alta para domicílio, podemos inferir que em 2010 a referenciação se reflectiu num aumento de 1718 dias de internamento e 1198 dias, em 2011. Estes resultados levantam algumas preocupações, uma vez que a implementação da $\mathrm{RNCCl}$ está praticamente concluída, não se prevendo um

\section{REFERÊNCIAS}

1. Portugal. Decreto-Lei n. ${ }^{\circ}$ 101/2006. DR 109 SÉRIE I-A de 2006-06-06.

2. Resolução do Conselho de Ministros n. 37 2010. DR 94 SÉRIE I de 2010-05-14

3. Rede Nacional para os Cuidados Continuados Integrados. Unidades de Internamento. [consultado 2012 Nov 15]. Disponível em: http://www. rncci.min-saude.pt/rncci/Paginas/ARede.aspx.

4. Rede Nacional para os Cuidados Continuados Integrados. Relatório de aumento de camas disponíveis, depois de 2013. Por outro lado, um aumento de dias de internamento em média de 1718 dias em 2010 e 1198 em 2011, sendo que apenas $21,4 \%$ e $23,5 \%$, respectivamente, foram referenciados traduz uma marcada incapacidade de resposta às necessidades actuais.

Este estudo apresenta algum viés, uma vez que o $\mathrm{mRS}$ utilizado, foi o da data de alta efectiva e não o do dia da referenciação, pelo que no intervalo do tempo, entre a referenciação e alta efectiva, houve nalgumas situações modificação do mRS. Dentro deste grupo de doentes encontramos aqueles com $\mathrm{mRS}$ de 6 , ou seja, falecidos, que se encontram no grupo de doentes referenciados. Estes representam os doentes que faleceram enquanto aguardavam vaga, muitas vezes de complicações infecciosas, mas cujo diagnóstico principal, se manteve como Acidente Vascular Cerebral.

\section{CONCLUSÃO}

A Implementação da Rede Nacional de Cuidados Continuados Integrados veio sem dúvida colmatar uma necessidade crescente da nossa população. Não nos podemos esquecer que o seu objectivo principal é promover a qualidade na prestação de cuidados de saúde e a sua continuidade na pós-alta hospitalar, passando também por facilitar o fluxo de doentes dos hospitais de agudos. Neste momento, apesar de apresentar uma melhoria relativamente ao ano de 2010, a capacidade de resposta é ainda manifestamente insuficiente. Perante esta incapacidade é imprescindível repensarmos estratégias, sendo importante equacionar o aumento de camas disponíveis na $\mathrm{RNCCl}$ e a necessidade de formação dos cuidadores informais, bem como da oferta de respostas, através de equipas multidisciplinares de apoio domiciliário, que permitam uma melhor e mais rápida capacidade na reintegração dos doentes na sua família.

\section{CONFLITOS DE INTERESSE}

Os autores declaram a inexistência de conflitos de interesse.

\section{FONTES DE FINANCIAMENTO}

O presente artigo não foi objecto de qualquer fonte externa de financiamento.

Apresentado previamente no Congresso Português de Neurologia 2012 - O Sono e os Sonhos, Novembro 2012, Lisboa.

monitorização do desenvolvimento e da actividade da Rede Nacional de Cuidados Continuados Integrados. Lisboa: RNCCl; 2011.

5. Rede Nacional para os Cuidados Continuados Integrados. Relatório de monitorização do desenvolvimento e da actividade da Rede Nacional de Cuidados Continuados Integrados. Lisboa: RNCCl; 2010.

6. Directiva Técnica $n^{\circ} 1$ UMCCI_2011_da Unidade de Missão para os Cuidados Continuados. 


\section{Rede Nacional de Cuidados Continuados e Tempo de Internamento dos Doentes com Acidente Vascular Cerebral 2010-2011 \\ Acta Med Port 2013:26:683-688}

Publicado pela Acta Médica Portuguesa, a Revista Científica da Ordem dos Médicos

Av. Almirante Gago Coutinho, 151

1749-084 Lisboa, Portugal.

Tel: +351218428 215

E-mail: submissao@actamedicaportuguesa.com

www.actamedicaportuguesa.com

ISSN:0870-399X | e-ISSN: 1646-0758

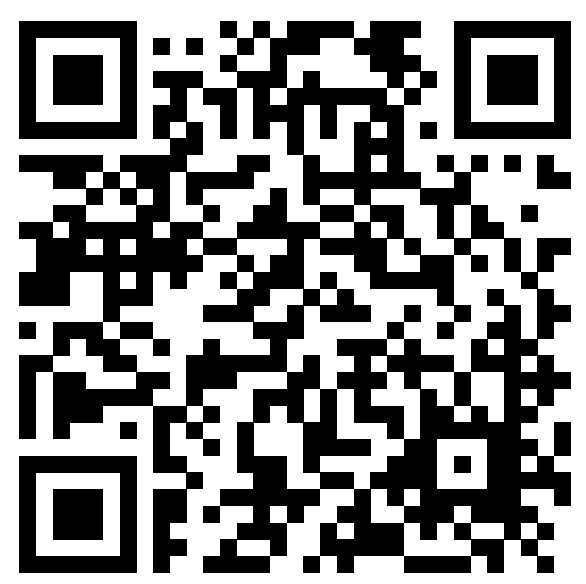

